\title{
FATIGUE INVESTIGATION OF THREADED PIPE CONNECTIONS
}

\author{
J. Van Wittenberghe ${ }^{1}$, J. De Pauw ${ }^{1}$, P. De Baets ${ }^{1}$, W. De Waele ${ }^{1}$, M. Abdel Wahab ${ }^{1}$, W. Ost ${ }^{1}$, \\ G. De Roeck ${ }^{2}$ and T.T. Bui ${ }^{2}$ \\ ${ }^{1}$ Ghent University, Laboratory Soete, Belgium \\ ${ }^{2}$ K.U.Leuven, Department of Civil Engineering, Belgium
}

\begin{abstract}
Threaded pipe connections are used to connect well casing, well tubing, drill pipes and risers. For many of these applications fatigue resistance plays an important role. In this study the fatigue properties of threaded connections are studied using a combination of finite element modelling and experimental testing. Using 2D axisymmetric FE analysis several connections are compared. It is shown that the load distribution over the engaged threads is an important feature. Experimental tests are carried out on three setups. A small scale four-point bending setup is used to develop S-N curves. An S-N curve for a standard API Line Pipe connection is compared to an S-N curve for a connection that showed an improved load distribution over the engaged threads in the FE analysis. On a medium scale four-point bending setup, strains together with crack opening are measured. The strain measurements are compared with the strains obtained by the numerical model. Finally a full scale resonant bending fatigue setup is presented, which will be used in future testing of pipe connections ranging from $168 \mathrm{~mm}$ (6") to $508 \mathrm{~mm}$ $(20 ")$ in diameter.
\end{abstract}

Keywords threaded connection, threaded and coupled, premium connection, pipe, finite element model, experiment, fatigue, four-point bending, resonant bending

\section{THREADED CONNECTIONS: AN OVERVIEW}

Fossil fuels such as oil and natural gas are finite resources. However, they will continue to be significant energy providers during the foreseeable future. Increased demand presents several engineering challenges since exploration of new sources moves further to greater depths, deeper ocean water and into harsher environments. This results in extreme requirements for the equipment used during exploration and exploitation of the wells.

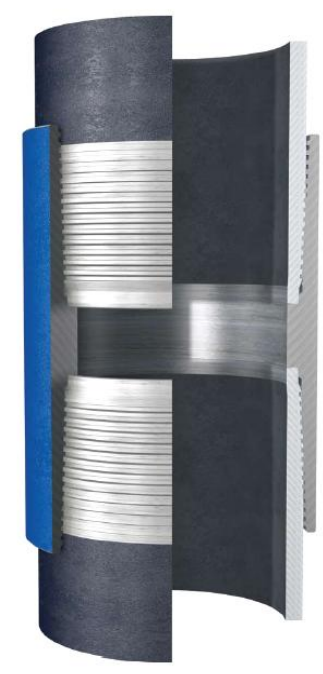

a

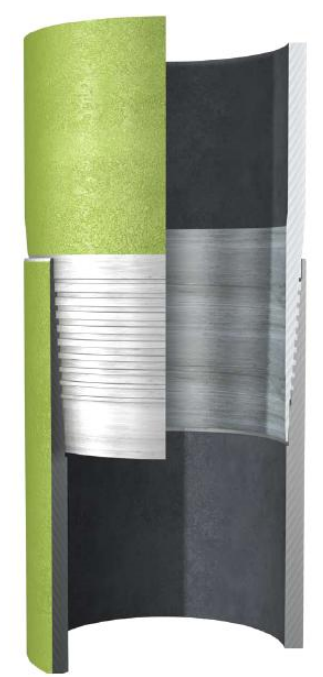

b

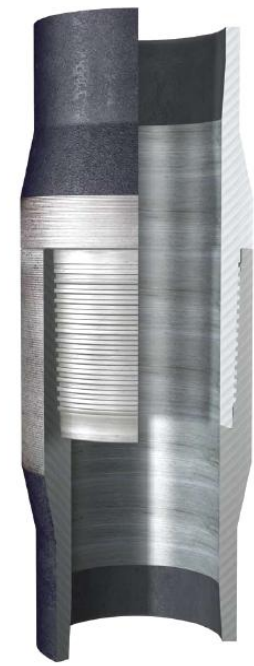

C

Figure 1. a) Threaded and Coupled, b) Integral Flush, c) Integral Upset Connection [1]

During well construction, a hole is drilled using a drilling tool connected to a long string of subsequent drill pipes. To avoid the well from collapsing, casing pipes are placed inside the well. After well completion, a tubing string is inserted by which the oil and gas flows to the surface. In case of offshore wells, an additional riser pipeline connects the wellhead at the bottom of the sea to a production platform. The drill pipes, the casing and tubing and in many cases also the riser pipes are connected using threaded connections. The threaded pipes of casing and tubing are regularly called Oil Country Tubular Goods (OCTG). Generally, threaded pipe connections can be divided in three different types, as can be seen in 
Figure 1 [1]. For the first type, the pipes carry male threads at both ends and a separate coupling part is used to connect them. These connections are called Threaded and Coupled (T\&C, see Figure 1.a) and are commonly used in OCTG and riser applications. The other types are called Integral types, since the pipes have a male and female part of the connection at either end. Hence no separate coupling part is used for these connections. When the connection is produced in the pipe material without any local increase in inside or outside diameter, it is called a flush connection (see Figure 1.b). However, when the connection is produced in a part with a thicker wall than the rest of the pipe, it is called an upset connection (see Figure 1.c). Integral flush connections are used in casing pipes while integral upset connections are more common for tubing and drill pipes. The male and female part of a threaded connection are called respectively pin and box.

A number of casing, tubing and drill pipe connections are standardized by the American Petroleum Institute (API) [2, 3]. Most OCTG connections are T\&C types, and the drill pipes have integral connections. However, as can be seen from Table 1, the applicability of those connections is restricted to oil wells and onshore gas wells with limited depth and bottom hole pressure. Once connections are needed that require better characteristics, so-called premium connections should be used, which are connections developed by private companies. Theses connections exhibit better performance than the standard API connections.

\begin{tabular}{|c|c|cc|}
\hline \multirow{2}{*}{ Gas Wells } & Offshore & \multicolumn{3}{|c|}{ Premium Connections } \\
\cline { 2 - 2 } & Onshore & \multirow{2}{*}{ API } & \\
\hline Oil Wells & & & \\
\hline Well Depths [km] & \multicolumn{2}{|c|}{3.0} & 6.0 \\
\hline Bottom Hole Pressure [bar] & \multicolumn{2}{|c|}{275} & 550 \\
\hline
\end{tabular}

Table 1. Applicability of API and premium connections

Typically standard OCTG connections were designed for static load cases, such as tension or compression, bending and internal or external pressure, that either alone or combined affect the string. The main design concern for these connections was leak-tightness. Riser connections are additionally subjected to high dynamic loads caused by waves and currents. And with drill strings dynamic loads are introduced by bending during drilling and torque vibrations. This introduces additional requirements for the fatigue resistance of these premium connections. Additionally, when moving to deeper wells and offshore gas wells, the bottom hole pressure increases and additional sealing measures have to be provided.

Although there exists a wide variety of premium drill connections, during the last years there is a tendency to use casing pipes for drilling particular wells [4-6]. This is more cost-effective since no drill pipes are necessary and the casing can stay in the well after drilling. However, this practice demands better fatigue properties for OCTG connections since they are subjected to additional dynamic loads due to the drilling operation. Hence with changing applications and environmental conditions, threaded connections of all kinds need continuous improvement and fatigue resistance is one of the important features that has to be considered.

In this study the fatigue resistance of threaded pipe connections is studied using numerical modelling and experimental testing. With the numerical model, a wide variety of connections can be assessed in a relatively fast and structured way, which can reveal general trends and underlying mechanisms. However experimental testing remains a necessity since the model output depends highly on prior assumptions, simplifications and boundary conditions. Hence experimental data serves as validation of the results generated by the simulations. In the following paragraphs an overview will be given of the obtained results of this study.

\section{NUMERICAL MODELLING}

\subsection{Axisymmetric model}

Threaded pipe connections are commonly modelled using 2D axisymmetric finite element models with elastic-plastic material behaviour and contact interaction of the threads [6-10]. Full 3D models of threaded couplings still require very long calculation times due to the high number of nodes in the contact analysis. The disadvantage of the 2D simulations is that they neglect the helical shape of the threads and the runout region. However, it was recently validated by the authors and earlier by Zhong [12] that the results of $2 \mathrm{D}$ axisymmetric models are in good agreement with the results of accurate time-consuming full 3D models.

The connection modelled in this study is a API Line Pipe threaded and coupled connection. The modelled connection is shown in Figure 2 and has a size of 4.5 " with dimensions according to the API 5B 
specifications [2]. For the numerical model, only the section in the dashed rectangle is used. The resulting model is shown in Figure 3. The model geometry is generated by the parametrical program ThreadGen ${ }^{\circ}$ developed at the Laboratory Soete [13]. This allows easy variation of the connection's geometrical parameters such as pin and box diameter and wall thickness, number of engaged threads and thread dimensions. This is particularly useful to compare different geometrical modifications, since numerous premium connections exist, which claim to provide better fatigue properties by changing the box geometry. However, no quantifiable results have been published to prove such claims.

\subsection{Analysis results}

To ensure adequate sealing and a robust connection while being subjected to external loads, the connections are preloaded. To introduce this preload, the API Line Pipe connection has a conical shape (see Figure 2). Premium connections frequently use rotary shouldered connections with one or more metalto-metal sealing surfaces. The connection is then assembled by giving it a number of rotations, which results in a certain make-up torque, which has a specific value for every connection type and size. The make-up is modelled in the 2D axisymmetric models by giving the pin and box a certain radial overlap corresponding to the specified number of make-up turns. Due to the combination of the make-up turns and external loads, together with the thread geometry, a complex multiaxial stress distribution develops over the connection. In Figure 4, the von Mises equivalent stress is shown for make-up only (Figure 4.a) and for the combination of make-up and an external axial tensile stress of $150 \mathrm{MPa}$ (Figure 4.b). Note that the location with the highest stress is the last engaged thread (LET) of the pin (as indicated by the arrow in Figure 4.b), which will be the region where fatigue cracks will tend to initiate. The high stress intensity at the LET of the pin is a result of the non-uniform load distribution over the different engaged threads of the connection (see Figure 5). For this reason and the fact that improved fatigue resistance is aimed for, one should try to get a more uniform load distribution over the connection.

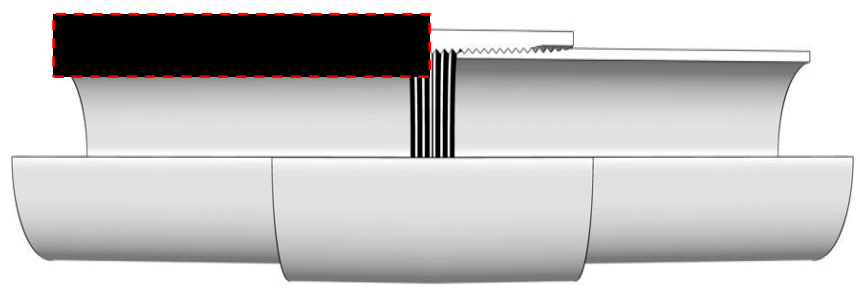

Figure 2. API Line Pipe T\&C connection: section view

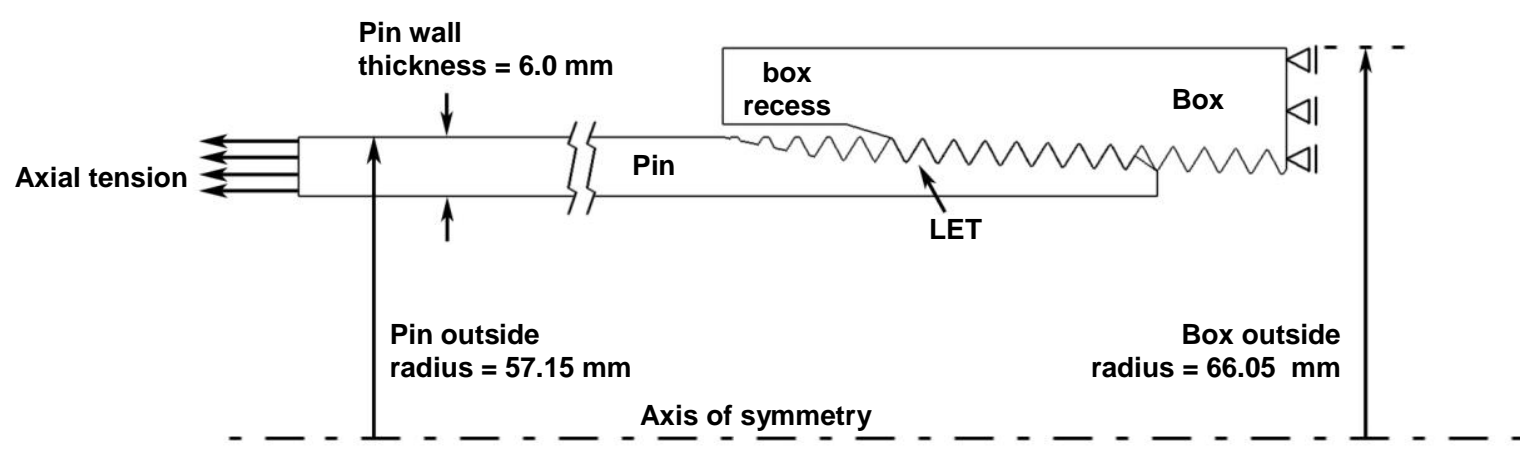

Figure 3. 2D axisymmetric model of the API Line Pipe connection
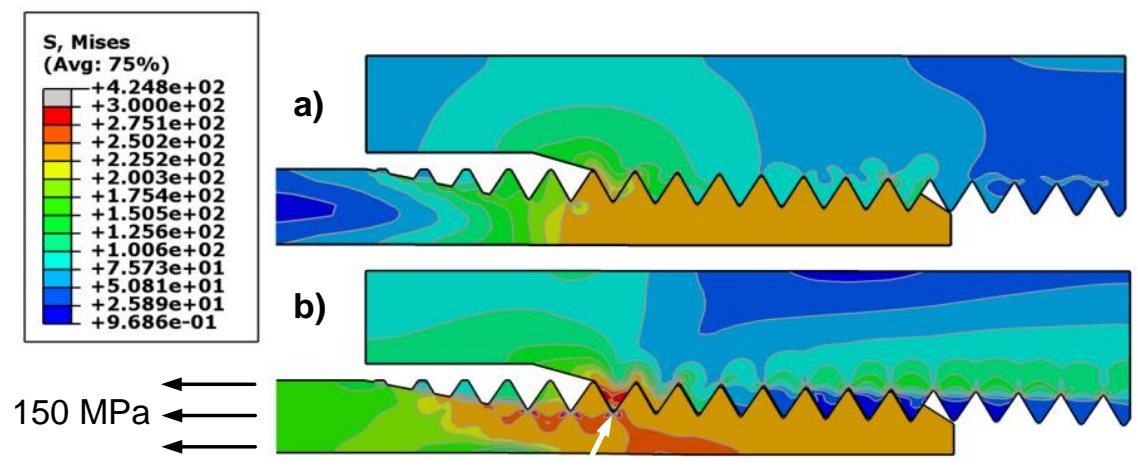

Figure 4.Von Mises stress distribution a) at make-up, b) with additional external axial tensile stress of $150 \mathrm{MPa}$ 


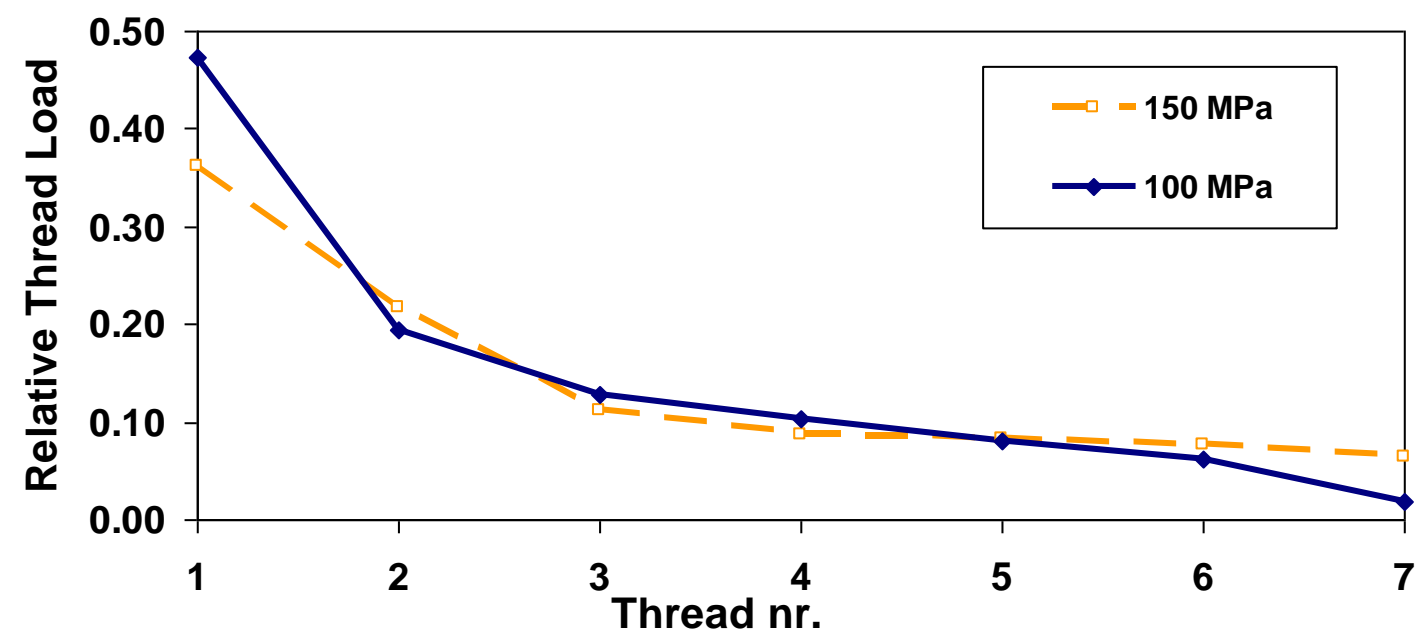

Figure 5. Load distribution over the engaged threads of the connection (LET = thread nr. 1)

\section{EXPERIMENTAL FATIGUE TEST SETUPS}

To obtain experimental results over a wide pipe diameter range and to be able to assess size effects, three different experimental fatigue setups are used. In the following paragraphs all three setups are discussed.

\subsection{Small scale four-point bending setup}

Tests were carried out on standard 1" (pipe diameter $=33.4 \mathrm{~mm}$ ) API Line Pipe connections on the small scale four-point bending setup to develop S-N curves. This setup, shown in Figure 6, is built on an ESH 100 $\mathrm{kN}$ universal test machine. The test sample consists of a central box (1a) with two made-up threaded pipes (1b) and is supported by the two outer load points (indicated by the blue arrows in the figure). A dynamic load is applied by the hydraulic actuator (2) and transferred through the loadcell (3) to a transverse beam that carries the two inner load points. To avoid a bending moment in the loadcell, the loadcell is connected to the transverse beam through a hinge (4).

Tests were carried out under load control with a load ratio $R=F_{\min } / F_{\max }=0.1$ and at a testing frequency of $15 \mathrm{~Hz}$. To detect through thickness cracks, the tube samples were pneumatically pressurized at a pressure between 1 and 2 bar. When a crack grows through the wall thickness of a pipe, pressure drops below a threshold and the test is stopped. The number of elapsed load cycles before the stop, is considered the fatigue life of the connection. To create two separate pressure chambers, a plug is inserted inside the box, between the two threaded pipe samples. This way a crack in either pipe sample could be distinguished from each other. The internal pressure in the two samples was continuously monitored using the pressure transducers (5) at both ends of the test sample. After a pressure drop, the connection was disassembled and the exact crack location was found using die penetrant. The failed pipe was then replaced by a dummy sample and the test continued until failure of the second pipe occurred.

In Figure 7.a the obtained S-N curve of the standard 1" API Line Pipe connection is compared to the results of an optimized connection for which the box wall thickness was reduced with $1 \mathrm{~mm}$. It can be seen that the latter has a fatigue limit that is about $20 \%$ higher. This better fatigue performance can be explained by the improved (i.e. more uniform) load distribution over the connection as shown in Figure 7.b. The stress amplitude $\sigma_{a}$ is given as a percentage of the material's yield stress $\sigma_{y}(356 \mathrm{MPa})$. 


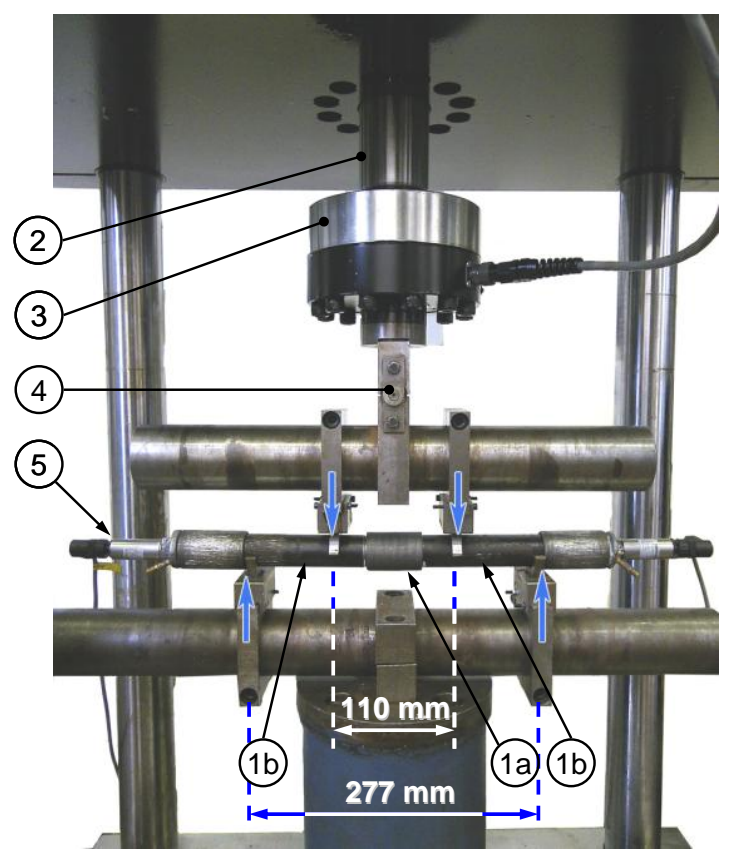

Figure 6. Small scale four-point bending test setup
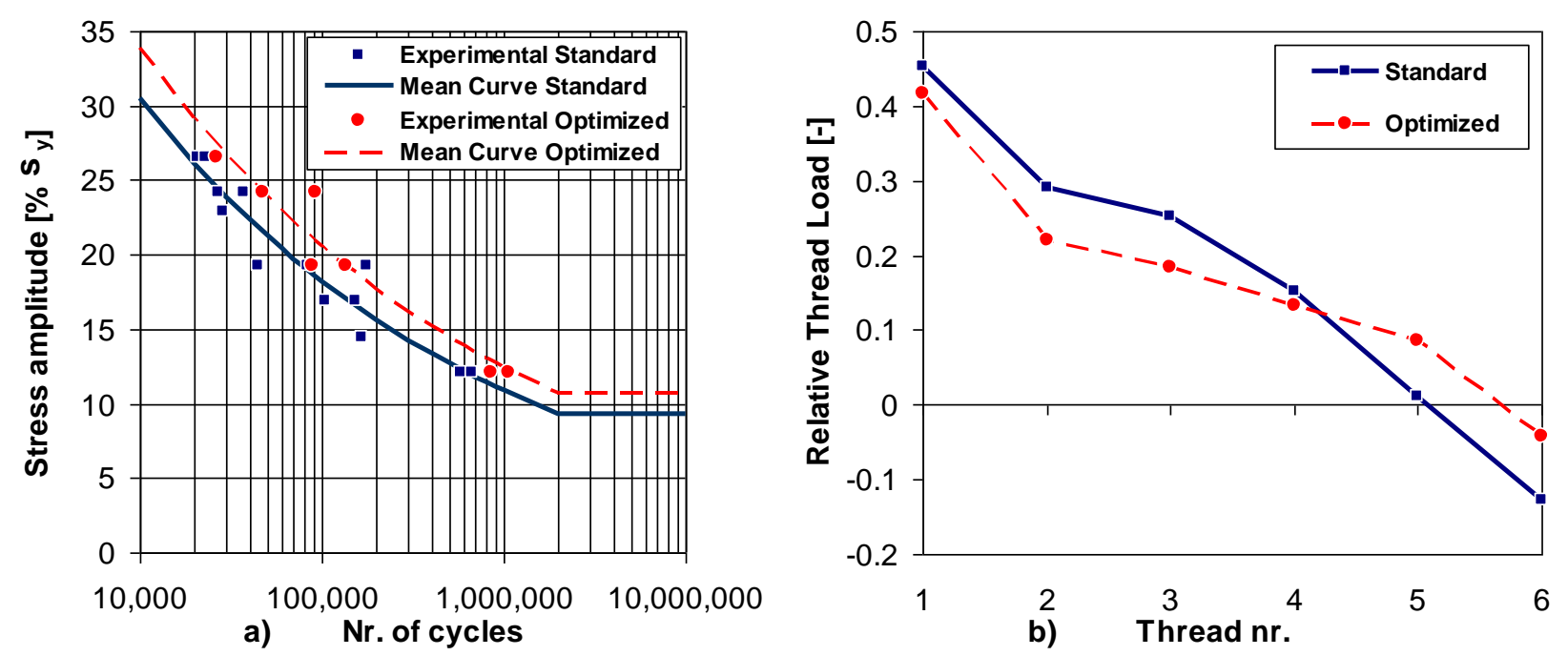

Figure 7. a) S-N curves for the standard 1" API Line Pipe connection and for a modified connection with changed wall thickness, b) thread load distribution for the standard and modified connection

\subsection{Medium scale four-point bending setup}

To test 4.5" (pipe diameter $=114.3 \mathrm{~mm}$ ) API Line Pipe Connections, the medium scale four-point bending test setup, schematically shown in Figure 8 , is used. The test specimen, consisting of two threaded pipe segments (1b) connected by a 4.5" API Line Pipe coupling (1a), is carried by two supports that are positioned $3 \mathrm{~m}$ apart on a central beam. A vertical force is applied by a hydraulic cylinder (2) that is connected to a transverse beam by a ball joint (4), with a loadcell (3) in between. The beam transmits the load to two points on the test specimen symmetric to the coupling. Strains are measured by strain gauges (5) on both pin and box. Since fatigue cracks are expected to initiate from the last engaged thread of the pin, two clip gauges (6) are mounted at either side of the box, to measure the axial distance between the edge of the box and a point on the pipe body of the pin. This way when a crack starts to grow, the crack opening displacement is detected by the clip gauges. Tests are carried out at a load frequency of $1 \mathrm{~Hz}$.

In Figure 9.a a comparison is given between the strains calculated by the finite element model as described in section 2 and the strains measured by the strain gauges. In Figure 9.b the crack opening displacement is shown as measured by the clip gauge over the side that cracked. It can be seen that the crack started to grow rapidly after about 18600 cycles. The test was stopped after 20350 cycles. 


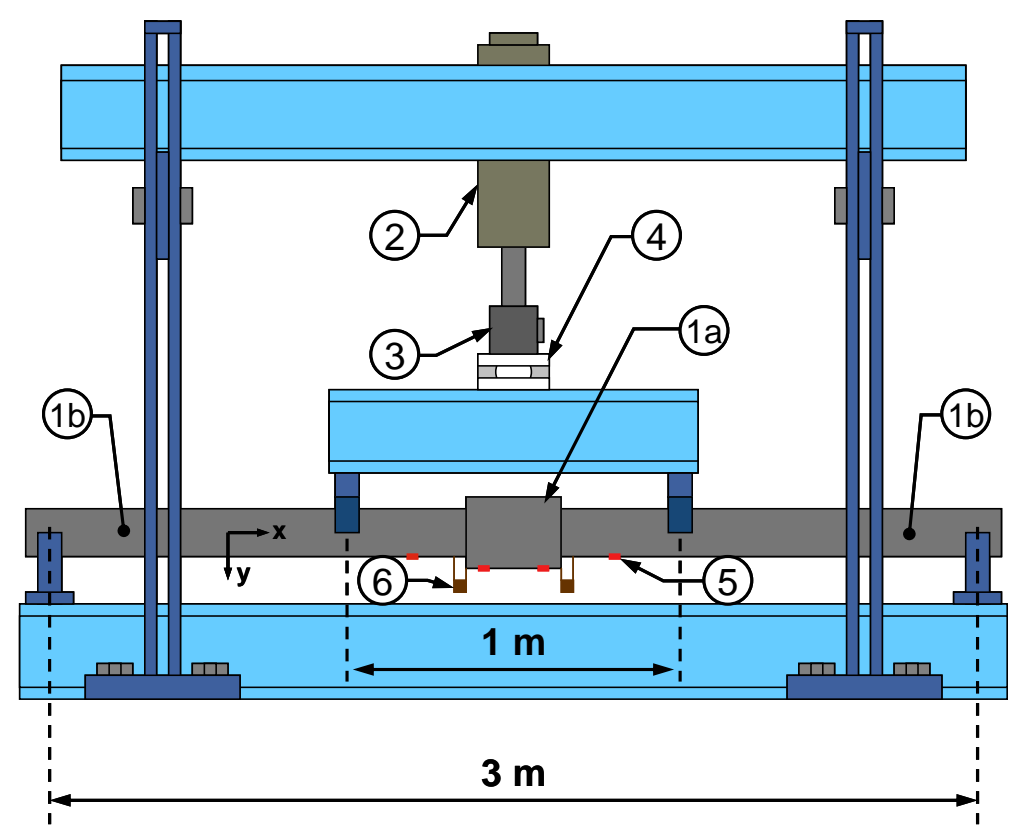

Figure 8. Medium scale four-point bending test setup
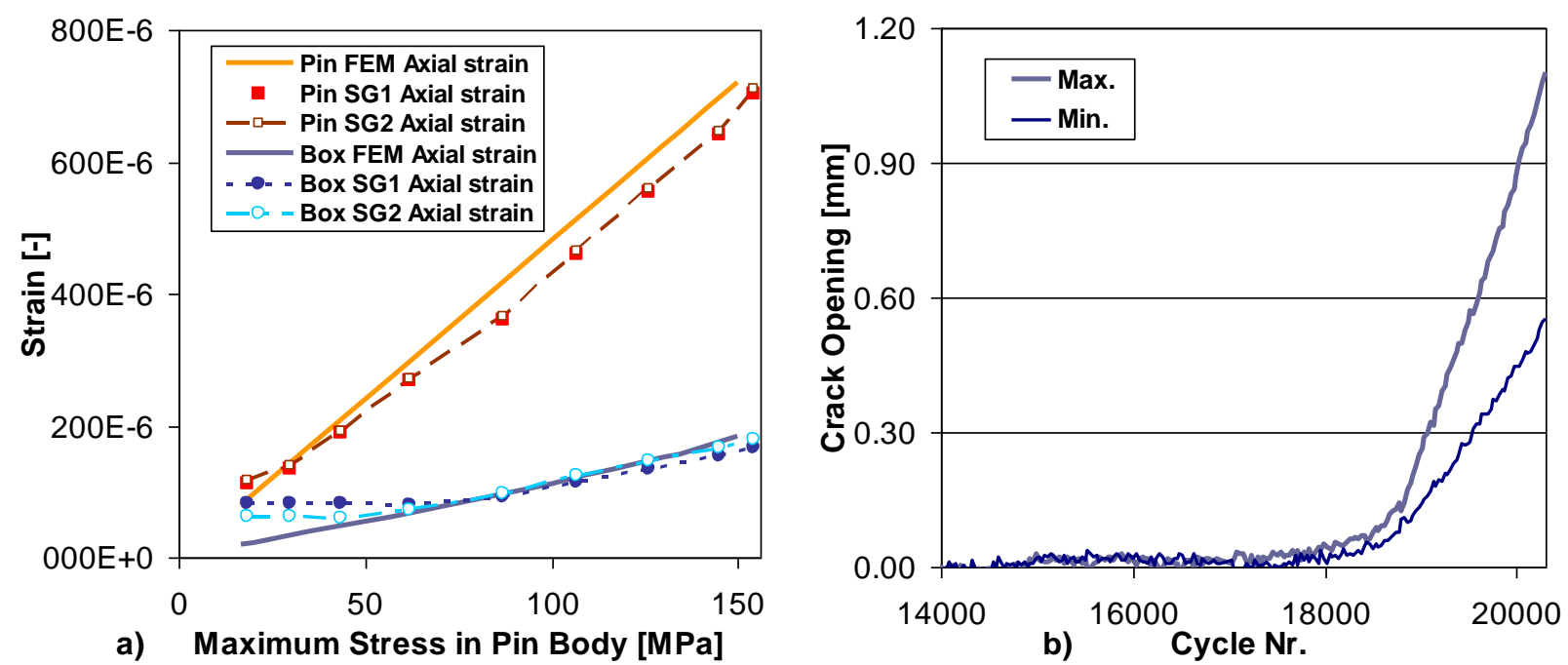

Figure 9. a) Comparison between modelled and measured strains, b) crack opening

\subsection{Full scale resonant bending fatigue setup}

To test couplings with a diameter up to 20 ", a new full scale resonant bending fatigue test setup was developed at the Laboratory Soete. A representation of the setup is shown in Figure 10. In this setup the test pipe (1) with a central threaded connection is excitated by a drive unit (2) with eccentric masses. This unit is powered via a cardan shaft (3) by an electrical motor (4) of $7.5 \mathrm{~kW}$. The excitation frequency is close to the natural frequency of the system. At the other pipe end a compensating mass (5) is attached to obtain a symmetric deformation of the pipe. The pipe is supported by the two supports (6) which are placed in the nodes of the first eigenmode of the system. The position of these supports can be changed by hydraulic cylinders. The total setup is placed on a heavy framework (7) and an additional safety frame (8) is placed over the central section of the pipe. This central section will be subjected to the highest cyclic loads during the fatigue test.

\begin{tabular}{|c|c|c|c|c|c|}
\hline Pipe Diameter & $\begin{array}{c}\text { Pipe Wall } \\
\text { Thickness }\end{array}$ & Pipe Length & Pipe Deflection & Frequency & $\begin{array}{c}\text { Motor } \\
\text { Power }\end{array}$ \\
\hline $\begin{array}{c}168 \mathrm{~mm} \text { to } 508 \mathrm{~mm} \\
(6 " \text { to } 20 ")\end{array}$ & $5 \mathrm{~mm}$ to $40 \mathrm{~mm}$ & $4 \mathrm{~m}$ to $6 \mathrm{~m}$ & $13 \mathrm{~mm}$ to $38 \mathrm{~mm}$ & $20 \mathrm{~Hz}$ to $40 \mathrm{~Hz}$ & $7.5 \mathrm{~kW}$ \\
\hline
\end{tabular}

Table 2. Resonant bending setup properties 
The main properties of the setup are summarized in Table 2. Based on the size of pipe diameter and wall thickness, the pipe length is chosen to obtain a natural frequency within the range of the electrical motor (from 20 to $40 \mathrm{~Hz}$ ). To lower the natural frequency of the pipe, it is filled with water which will be slightly pressurized for the purpose of leak-detection. The deflection of the pipe depends on the desired stress level and will be about 13 to $38 \mathrm{~mm}$. Test results obtained with this new setup will be published in the future.

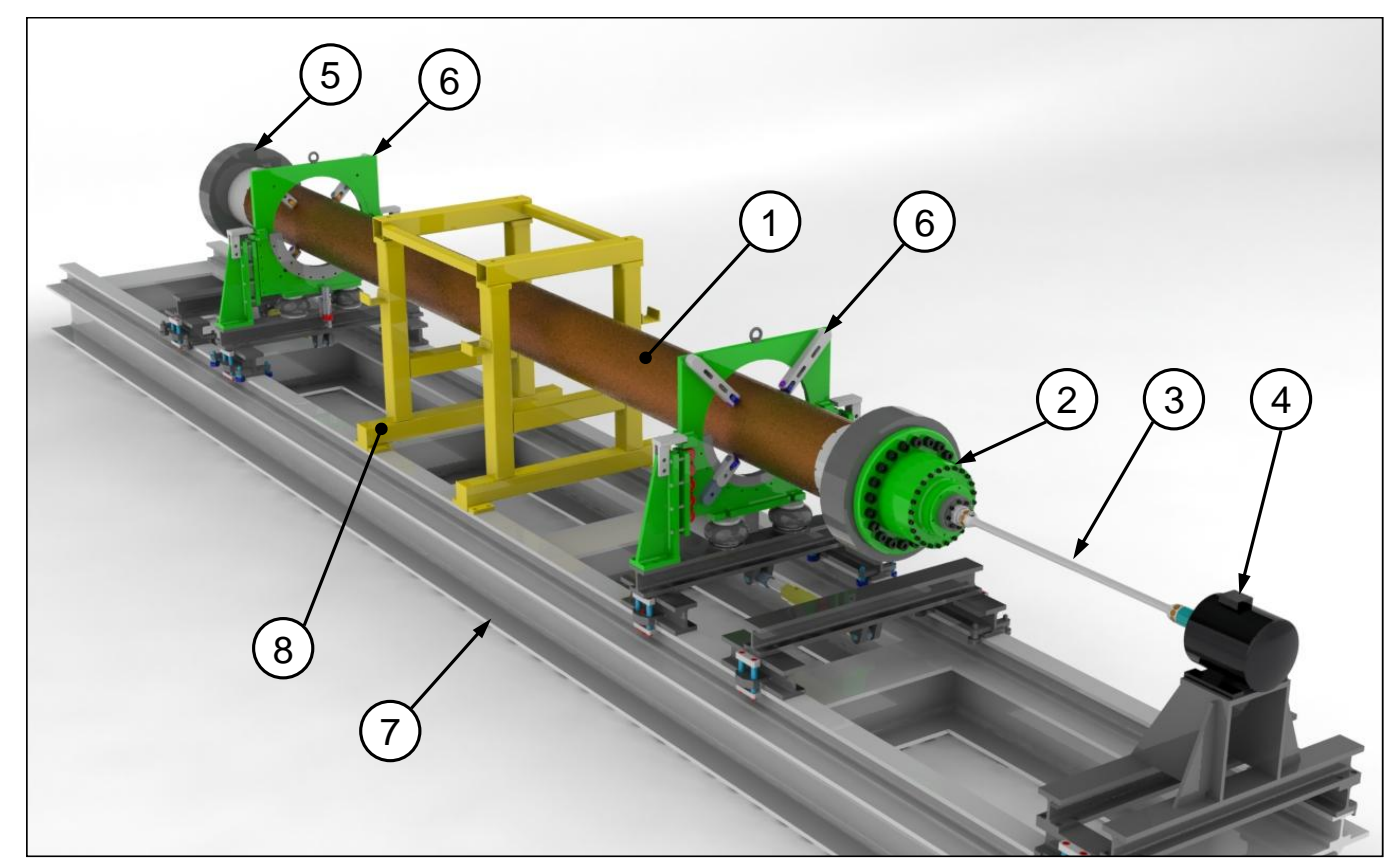

Figure 10. Full scale resonant bending fatigue setup

\section{CONCLUSIONS}

Fatigue resistance is an important feature for a wide range of threaded pipe connections. In this paper the fatigue properties of threaded connections are studied using a combination of numerical modelling and experimental testing. From the developed 2D axisymmetric finite element model it is known that the high stress concentration at the LET of the pin is caused by a highly non-uniform load distribution over the different engaged threads of the connection. Three different experimental fatigue setups for testing pipes from small to full scale are discussed. Results of strain measurements and S-N curves correspond well with trends observed in the numerical model. Future work will include full scale tests on a resonant bending fatigue setup.

\section{ACKNOWLEDGEMENTS}

The authors would like to acknowledge the financial support of the BOF fund (B/04939) of the Ghent University and of the FWO Vlaanderen (3G022806).

\section{REFERENCES}

[1] TenarisHydril, Premium Connections Catalogue, http://www.tenaris.com/tenarishydril/, Version 01, March 2009.

[2] API Specification 5B, Specification for Threading, Gauging and Thread Inspection of Casing, Tubing and Line Pipe Threads (U.S. Customary Units), American Petroleum Institute, fourteenth edition, 1996.

[3] API Specification 7, Specification for Rotary Drill Stem Elements, American Petroleum Institute.

[4] Santi, N. J., Carcagno, G. E., Toscano, R., Premium \& Semi-premium Connections Design Optimization for Varied Drilling-with-Casing Applications, Offshore Technology Conference, OTC17221, 2005.

[5] Strickler, R.D., Wadsworth, T.M., Drilling with casing: Are you damaging your casing?, World Oil, 3, 5153, 2005.

[6] Carcagno, G.E., Bufalini, A., Conde, L., Toscano, R., Low SCF Integral Premium Connections for Use in Highly Demanding Casing and Tubing Drilling Operations, Offshore Technology Conference, OTC16566, 2004. 
[7] Dvorkin, E.N. and Toscano, R.G., Finite element models in the steel industry, Part II: Analyses of tubular products performance, Computers and Structures, 81(8-11), 575-594, 2003.

[8] Kristensen, A.S., Toor, K., Solem, S.I., Finite Element analysis of jar connections: modeling considerations, Journal of Structural Mechanics: Special issue for the 18th Nordic Seminar on Computational Mechanics, 1-4, Helsinki, Finland, 2005.

[9] Guangjie, Y., Zhenqiang, Y., Qinghua, W., Zhentong, T., Numerical and experimental distribution of temperature and stress fields in API round threaded connection, Engineering Failure Analysis, 13(8), 1275-1284, 2006.

[10]Bertini, L., Beghini, M., Santus, C. and Baryshnikov A., Fatigue on drill string conical threaded connections, test results and simulations, 9th Int. Fatigue Congress, Atlanta, USA, 2006.

[11] Santus, C., Bertini, L., Beghini, M., Merlo, A., Baryshnikov, A., Torsional strength comparison between two assembling techniques for aluminium drill pipe to steel tool joint connection, International Journal of Pressure Vessels and Piping, 86(2-3), 177-186, 2009.

[12]Zhong, A., Thread Connection Response to Critical Pressures, Abaqus Users' Conference, pp. 690706, Paris, France, May 2007.

[13] Van Wittenberghe, J., De Baets, P., De Waele W., Modeling of preloaded threaded pipe connections, 8th National Congres on Theoretical and Applied Mechanics, 149-156, Brussels, Belgium, 2009. 